\title{
NEUMOTÓRAX BILATERAL ESPONTÁNEO ASOCIADO A QUIMIOTERAPIA
}

\author{
SPONTANEOUS BILATERAL PNEUMOTHORAX ASSOCIATED WITH \\ CHEMOTHERAPY
}

Jorge Luna-Abanto

PRESENTACIÓN DEL CASO

1 Instituto Nacional de Enfermedades Neoplásicas. Lima, Perú.

Escuela de Posgrado, Universidad Peruana Cayetano Heredia. Lima,

Perú.

Correspondencia a:

Paciente varón de 42 años natural y procedente de Lima, sin antecedentes médicos de importancia, con diagnóstico de Meduloblastoma Cerebeloso operado en el 2005 (craneotomía suboccipital izquierda + resección total de tumor), recibió adyuvancia con radioterapia y quimioterapia la cual consistió en 10 cursos de Carmustina (BCNU) y Vincristina con regular tolerancia. Acude a control en Agosto 2016, refirió tiempo de enfermedad de 3 meses caracterizado por disnea a grandes esfuerzos la cual empeora súbitamente en la última semana. Al examen físico: paciente hemodinámicamente estable, taquicárdico, saturación de oxígeno $92 \%$. La radiografía de tórax frontal muestra neumotórax bilateral a predominio izquierdo y signos sugestivos de fibrosis pulmonar.

El manejo inicial consistió en colocación de drenaje pleural izquierdo a succión continua durante 15 días, al no evidenciar mejoría clínico radiológica, el paciente fue sometido a toracotomía exploradora izquierda. Durante el procedimiento se evidenció la presencia de bulas subpleurales múltiples, adherencias pleuromediastinales y del lóbulo inferior a diafragma, el parénquima pulmonar fue fibroso y se logró la expansión del lóbulo inferior, língula, el lóbulo superior expande parcialmente. Se realizó pleurodesis con talco hemostático y colocó drenaje pleural bilateral. En el postoperatorio el paciente tuvo manejo en unidad crítica por inestabilidad hemodinámica, fibrilación auricular e insuficiencia cardiaca, falleciendo 2 días después. \\ INTRODUCCIÓN}

El neumotórax bilateral espontáneo es una entidad rara, se asocia con enfermedades pulmonares de base, histiocitosis de células de Langerhans, linfangioleiomiomatosis, sarcoma metastásico y tuberculosis miliar, así como la variante anatómica "tórax de búfalo", caracterizada por la presencia de una comunicación (natural o adquirida) entre las dos cavidades pleurales ${ }^{1,2}$. Las enfermedades pulmonares son comunes en pacientes con cáncer que recibieron quimioterapia, sin embargo es difícil predecir la toxicidad pulmonar por estos agentes previo al tratamiento. Además, la presencia de fibrosis pulmonar y neumotórax bilateral es poco frecuente en estos pacientes ${ }^{3}$. El manejo del neumotórax bilateral se basa en la colocación de sistemas de drenaje pleural, así como pleurodesis química, la cual ha demostrado seguridad y efectividad. La videotoracoscopia y bulectomia está indicada en pacientes con neumotórax espontáneo recurrente 4 . 
Figura 1. Radiografía de tórax frontal: Neumotórax bilateral a predominio izquierdo (30\%) asociado a signos de fibrosis pulmonar

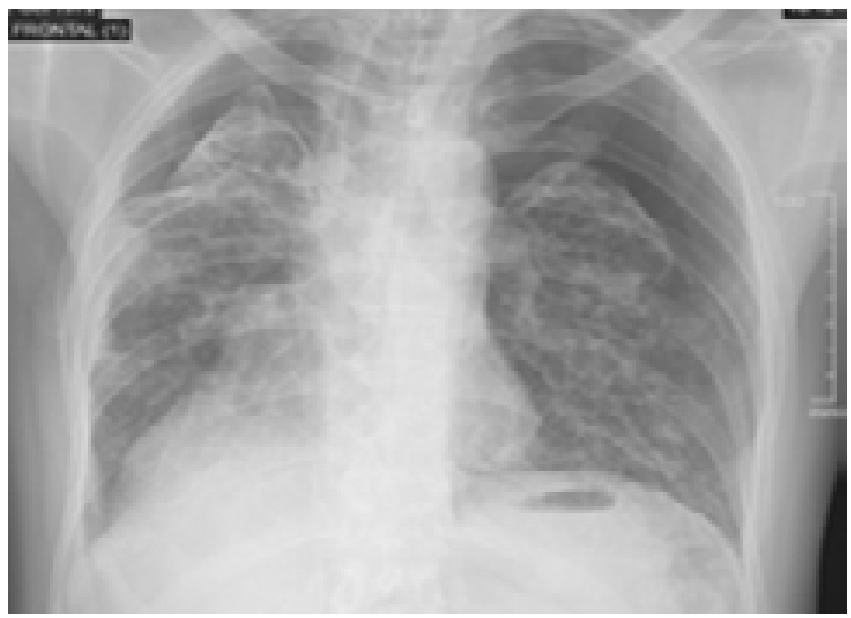

Figura 2. Tomografía de tórax sin contraste: Neumotórax bilateral a predominio derecho, presencia de tubo de drenaje pleural en hemitórax izquierdo, cambios intersticiales en ambos pulmones, presencia de enfisema subcutáneo en pared torácica izquierda y región axilar ipsilateral.

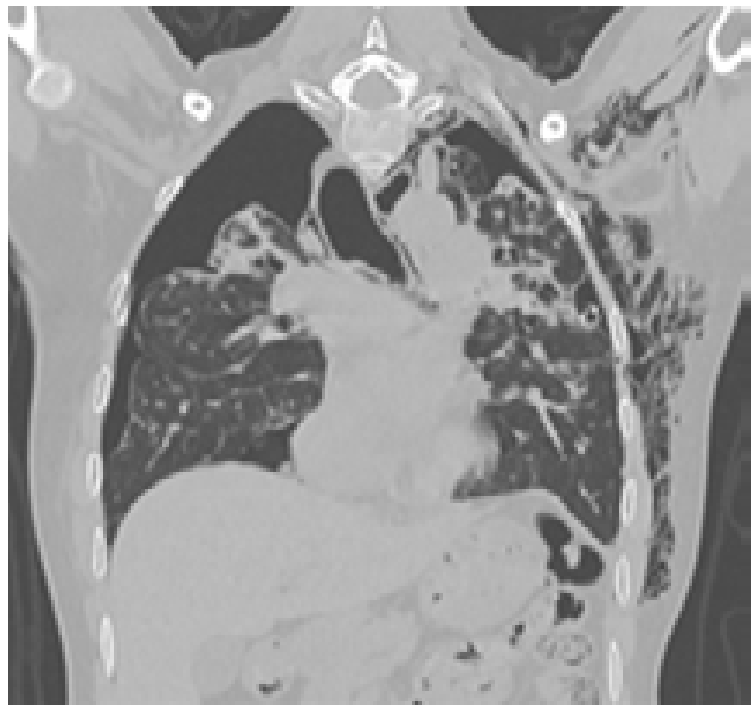

1.Albores J, Abtin F, Barjaktarevic I. A 44-year-old man with bilateral pneumothorax. Chest. 2015 Oct;148(4):e118-e121.

2.Saenz A, Balestra R. Bilateral Spontaneous Pneumothoraces in a Healthy Young Adult. Am J Respir Crit Care Med. 2011 Dec 15;184(12):e3.

3.Fang L, Huang CJ, Chuang AY, Tan TD, Liu CC. Chemotherapyrelated delayed bilateral spontaneous pneumothorax and lung fibrosis: methotrexate or cyclophosphamide, or both?. Clin Respir J. 2010 Oct;4(4):254-5.

4.How $\mathrm{CH}$, Hsu HH, Chen JS. Chemical pleurodesis for spontaneous pneumothorax. J Formos Med Assoc. 2013 Dec;112(12):749-55. 